\title{
Determination of the $\Lambda_{c}^{+}$spin via the reaction $e^{+} e^{-} \rightarrow \Lambda_{c} \bar{\Lambda}_{c}$
}

M. Ablikim, ${ }^{1}$ M. N. Achasov, ${ }^{10, c}$ P. Adlarson,${ }^{67}$ S. Ahmed,${ }^{15}$ M. Albrecht, ${ }^{4}$ R. Aliberti, ${ }^{28}$ A. Amoroso, ${ }^{66 a, 66 c}$ M. R. An,${ }^{32}$ Q. An ${ }^{63,49}$ X. H. Bai,${ }^{57}$ Y. Bai, ${ }^{48}$ O. Bakina,${ }^{29}$ R. Baldini Ferroli, ${ }^{23 a}$ I. Balossino, ${ }^{24 a}$ Y. Ban, ${ }^{38, k}$ K. Begzsuren,${ }^{26}$ N. Berger,${ }^{28}$ M. Bertani, ${ }^{23 a}$ D. Bettoni, ${ }^{24 a}$ F. Bianchi ${ }^{66 a, 66 c}$ J. Bloms, ${ }^{60}$ A. Bortone, ${ }^{66 a, 66 c}$ I. Boyko, ${ }^{29}$ R. A. Briere, ${ }^{5}$ H. Cai, ${ }^{68}$ X. Cai,${ }^{1,49}$ A. Calcaterra, ${ }^{23 a}$ G. F. Cao ${ }^{1,54}$ N. Cao, ${ }^{1,54}$ S. A. Cetin, ${ }^{53 a}$ J. F. Chang, ${ }^{1,49}$ W. L. Chang, ${ }^{1,54}$ G. Chelkov, ${ }^{29, b}$ D. Y. Chen, ${ }^{6}$ G. Chen, ${ }^{1}$ H. S. Chen, ${ }^{1,54}$ M. L. Chen, ${ }^{1,49}$ S. J. Chen, ${ }^{35}$ X. R. Chen, ${ }^{25}$ Y. B. Chen, ${ }^{1,49}$ Z. J. Chen, ${ }^{20,1}$ W. S. Cheng, ${ }^{66 c}$ G. Cibinetto, ${ }^{24}$ F. Cossio, ${ }^{66 c}$ X. F. Cui, ${ }^{36}$ H. L. Dai, ${ }^{1,49}$ X. C. Dai,,${ }^{1,54}$ A. Dbeyssi, ${ }^{15}$ R. E. de Boer, ${ }^{4}$ D. Dedovich, ${ }^{29}$ Z. Y. Deng, ${ }^{1}$ A. Denig, ${ }^{28}$ I. Denysenko, ${ }^{29}$ M. Destefanis, ${ }^{66,66 \mathrm{c}}$ F. De Mori, ${ }^{66 a, 66 \mathrm{c}}$ Y. Ding ${ }^{33}{ }_{1,5}$ C. Dong, ${ }^{36}$ J. Dong, ${ }^{1,49}$ L. Y. Dong, ${ }^{1,54}$ M. Y. Dong, ${ }^{1,49,54}$ X. Dong, ${ }^{68}$ S. X. Du, ${ }^{71}$ Y. L. Fan ${ }^{68}$ J. Fang, ${ }^{1,49}$ S. S. Fang, ${ }^{1,54}$ Y. Fang, ${ }^{1}$ R. Farinelli, ${ }^{24 a}$ L. Fava, ${ }^{66,66 c}$ F. Feldbauer, ${ }^{4}$ G. Felici, ${ }^{23 a}{ }^{2}$ C. Q. Feng, ${ }^{63,49}$ J. H. Feng, ${ }^{50}$ M. Fritsch, ${ }^{4}$ C. D. Fu, ${ }^{1}$ Y. Gao, ${ }^{63,49}$ Y. Gao, ${ }^{38, k}$ Y. Gao, ${ }^{64}$ Y. G. Gao, ${ }^{6}$ I. Garzia, ${ }^{24 a, 24 b}$ P. T. Ge, ${ }^{68}$ C. Geng, ${ }^{50}$ E. M. Gersabeck, ${ }^{58}$ A. Gilman, ${ }^{61}$ K. Goetzen, ${ }^{11}$ L. Gong, ${ }^{33}$ W. X. Gong, ${ }^{1,49}$ W. Gradl ${ }^{28}$ M. Greco, ${ }^{66 a, 66 c}$ L. M. Gu, ${ }^{35}$ M. H. Gu ${ }^{1,49}$ S. Gu, ${ }^{2}$ Y. T. Gu, ${ }^{13}$ C. Y. Guan, ${ }^{1,54}$ A. Q. Guo, ${ }^{22}$ L. B. Guo ${ }^{34}$ R. P. Guo, ${ }^{40}$ Y. P. Guo, ${ }^{9, \mathrm{~h}}$ A. Guskov, ${ }^{29, b}$ T. T. Han,${ }^{41}$ W. Y. Han,${ }^{32}$ X. Q. Hao ${ }^{16}$ F. A. Harris ${ }^{56}$ K. L. He, ${ }^{1,54}$ F. H. Heinsius, ${ }^{4}$ C. H. Heinz, ${ }^{28}$ T. Held, ${ }^{4}$ Y. K. Heng, ${ }^{1,49,54}$ C. Herold, ${ }^{51}$ M. Himmelreich, ${ }^{11, f}$ T. Holtmann, ${ }^{4}$ G. Y. Hou, ${ }^{1,54}$ Y. R. Hou,${ }^{54}$ Z. L. Hou, ${ }^{1}$ H. M. Hu, ${ }^{1,54}$ J. F. Hu, ${ }^{47, m}$ T. Hu, ${ }^{1,49,54}$ Y. Hu, ${ }^{1}$ G. S. Huang, ${ }^{63,49}$ L. Q. Huang, ${ }^{64}$ X. T. Huang, ${ }^{41}$ Y. P. Huang, ${ }^{1}$ Z. Huang, ${ }^{38, k}$ T. Hussain, ${ }^{65}$ N. Hüsken, ${ }^{22,28}$ W. Ikegami Andersson, ${ }^{67}$ W. Imoehl,${ }^{22}$ M. Irshad, ${ }^{63,49}$ S. Jaeger, ${ }^{4}$ S. Janchiv, ${ }^{26, j}$ Q. Ji, ${ }^{1}$ Q. P. Ji ${ }^{16}$ X. B. Ji, ${ }^{1,54}$ X. L. Ji, ${ }^{1,49}$ Y. Y. Ji, ${ }^{41}$ H. B. Jiang ${ }^{41}$ X. S. Jiang,${ }^{1,49,54}$ J. B. Jiao, ${ }^{41}$ Z. Jiao, ${ }^{18}$ S. Jin, ${ }^{35}$ Y. Jin, ${ }^{57}$ M. Q. Jing, ${ }^{1,54}$ T. Johansson, ${ }^{67}$ N. Kalantar-Nayestanaki, ${ }^{55}$ X. S. Kang, ${ }^{33}$ R. Kappert, ${ }^{55}$ M. Kavatsyuk ${ }^{55}$ B. C. Ke, ${ }^{43,1}$ I. K. Keshk, ${ }^{4}$ A. Khoukaz ${ }^{60}$ P. Kiese, ${ }^{28}$ R. Kiuchi, ${ }^{1}$ R. Kliemt,${ }^{11}$ L. Koch, ${ }^{30}$ O. B. Kolcu, ${ }^{53 a, e}$ B. Kopf, ${ }^{4}$ M. Kuemmel, ${ }^{4}$ M. Kuessner, ${ }^{4}$ A. Kupsc, ${ }^{67}$ M. G. Kurth, ${ }^{1,54}$ W. Kühn, ${ }^{30}$ J. J. Lane, ${ }^{58}$ J. S. Lange, ${ }^{30}$ P. Larin, ${ }^{15}$ A. Lavania, ${ }^{21}$ L. Lavezzi, ${ }^{66 a, 66 c}$ Z. H. Lei, ${ }^{63,49}$ H. Leithoff, ${ }^{28}$ M. Lellmann, ${ }^{28}$ T. Lenz, ${ }^{28}$ C. Li,${ }^{39}$ C. H. Li, ${ }^{32}$ Cheng Li, ${ }^{63,49}$ D. M. Li ${ }^{71}$

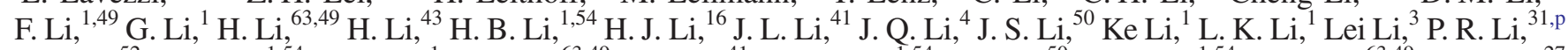
S. Y. Li, ${ }^{52}$ W. D. Li, ${ }^{1,54}$ W. G. Li,${ }^{1}$ X. H. Li ${ }^{63,49}$ X. L. Li ${ }^{41}$ Xiaoyu Li,${ }^{1,54}$ Z. Y. Li,${ }^{50}$ H. Liang, ${ }^{1,54}$ H. Liang, ${ }^{63,49}$ H. Liang, ${ }^{27}$ Y. F. Liang, ${ }^{45}$ Y. T. Liang, ${ }^{25}$ G. R. Liao, ${ }^{12}$ L. Z. Liao,,${ }^{1,54}$ J. Libby, ${ }^{21}$ C. X. Lin, ${ }^{50}$ B. J. Liu, ${ }^{1}$ C. X. Liu, ${ }^{1}$ D. Liu, ${ }^{15,63}$ F. H. Liu ${ }^{44}$ Fang Liu, ${ }^{1}$ Feng Liu, ${ }^{6}$ H. B. Liu, ${ }^{13}$ H. M. Liu, ${ }^{1,54}$ Huanhuan Liu, ${ }^{1}$ Huihui Liu, ${ }^{17}$ J. B. Liu, ${ }^{63,49}$ J. L. Liu, ${ }^{64}$ J. Y. Liu,${ }^{1,54}$

K. Liu, ${ }^{1}$ K. Y. Liu, ${ }^{33}$ L. Liu, ${ }^{63,49}$ M. H. Liu, ${ }^{9, h}$ P. L. Liu, ${ }^{1}$ Q. Liu ${ }^{68}$ Q. Liu, ${ }^{54}$ S. B. Liu, ${ }^{63,49}$ Shuai Liu, ${ }^{46}$ T. Liu, ${ }^{1,54}$ W. M. Liu, ${ }^{63,49}$ X. Liu, ${ }^{31, n, o}$ Y. Liu, ${ }^{31, n, o}$ Y. B. Liu, ${ }^{36}$ Z. A. Liu, ${ }^{1,49,54}$ Z. Q. Liu, ${ }^{41}$ X. C. Lou, ${ }^{1,49,54}$ F. X. Lu, ${ }^{50}$ H. J. Lu, ${ }^{18}$ J. D. Lu, ${ }^{1,54}$ J. G. Lu, ${ }^{1,49}$ X. L. Lu, ${ }^{1}$ Y. Lu, ${ }^{1}$ Y. P. Lu,${ }^{1,49}$ C. L. Luo, ${ }^{34}$ M. X. Luo, ${ }^{70}$ P. W. Luo, ${ }^{50}$ T. Luo,${ }^{9, h}$ X. L. Luo,, 49

X. R. Lyu ${ }^{54}$ F. C. Ma ${ }^{33}$ H. L. Ma, ${ }^{1}$ L. L. Ma, ${ }^{41}$ M. M. Ma, ${ }^{1,54}$ Q. M. Ma, ${ }^{1}$ R. Q. Ma ${ }^{1,54}$ R. T. Ma,${ }^{54}$ X. X. Ma,${ }^{1,54}$

X. Y. Ma ${ }^{1,49}$ F. E. Maas, ${ }^{15}$ M. Maggiora, ${ }^{66 a, 66 c}$ S. Maldaner, ${ }^{4}$ S. Malde ${ }^{61}$ Q. A. Malik ${ }^{65}$ A. Mangoni, ${ }^{23 b}$ Y. J. Mao, ${ }^{38, k}$ Z. P. Mao, ${ }^{1}$ S. Marcello, ${ }^{66 a, 66 c}$ Z. X. Meng, ${ }^{57}$ J. G. Messchendorp,${ }^{55}$ G. Mezzadri, ${ }^{24 a}$ T. J. Min, ${ }^{35}$ R. E. Mitchell, ${ }^{22}$ X. H. Mo, ${ }^{1,49,54}$ Y. J. Mo, ${ }^{6}$ N. Yu. Muchnoi, ${ }^{10, \mathrm{c}}$ H. Muramatsu, ${ }^{59}$ S. Nakhoul, ${ }^{11, f}$ Y. Nefedov, ${ }^{29}$ F. Nerling,,${ }^{11, f^{\prime}}$

I. B. Nikolaev, ${ }^{10, \mathrm{c}}$ Z. Ning, ${ }^{1,49}$ S. Nisar, ${ }^{8, \mathrm{i}}$ S. L. Olsen, ${ }^{54}$ Q. Ouyang, ${ }^{1,49,54}$ S. Pacetti, ${ }^{23 b, 23 \mathrm{c}}$ X. Pan, ${ }^{9, \mathrm{~h}}$ Y. Pan, ${ }^{58}$ A. Pathak, ${ }^{1}$ P. Patteri, ${ }^{23 a}$ M. Pelizaeus, ${ }^{4}$ H. P. Peng, ${ }^{63,49}$ K. Peters,${ }^{11, f}$ J. Pettersson, ${ }^{67}$ J. L. Ping, ${ }^{34}$ R. G. Ping $\odot,{ }^{1,54}$ R. Poling, ${ }^{59}$ V. Prasad ${ }^{63,49}$ H. Qi ${ }^{63,49}$ H. R. Qi ${ }^{52}$ K. H. Qi ${ }^{25}$ M. Qi ${ }^{35}$ T. Y. Qi ${ }^{9}{ }^{3}$ S. Qian, ${ }^{1,49}$ W. B. Qian, ${ }^{54}$ Z. Qian, ${ }^{50}$ C. F. Qiao, ${ }^{54}$ L. Q. Qin, ${ }^{12}$ X. P. Qin, ${ }^{9}$ X. S. Qin, ${ }^{41}$ Z. H. Qin,,${ }^{1,49}$ J. F. Qiu, ${ }^{1}$ S. Q. Qu, ${ }^{36}$ K. H. Rashid, ${ }^{65}$ K. Ravindran, ${ }^{21}$ C. F. Redmer, ${ }^{28}$ A. Rivetti, ${ }^{66 c}$ V. Rodin, ${ }^{55}$ M. Rolo ${ }^{66 c}$ G. Rong,,${ }^{1,54}$ Ch. Rosner, ${ }^{15}$ M. Rump, ${ }^{60}$ H. S. Sang, ${ }^{63}$ A. Sarantsev, ${ }^{29, d}$ Y. Schelhaas, ${ }^{28}$ C. Schnier, ${ }^{4}$ K. Schoenning, ${ }^{67}$ M. Scodeggio, ${ }^{24 a, 24 b}$ D. C. Shan, ${ }^{46}$ W. Shan ${ }^{19}$ X. Y. Shan, ${ }^{63,49}$ J. F. Shangguan, ${ }^{46}$ M. Shao, ${ }^{63,49}$ C. P. Shen, ${ }^{9}$ H. F. Shen, ${ }^{1,54}$ P. X. Shen,${ }^{36}$ X. Y. Shen, ${ }^{1,54}$ H. C. Shi ${ }^{63,49}$ R. S. Shi, ${ }^{1,54}$ X. Shi, ${ }^{1,49}$ X. D. Shi, ${ }^{63,49}$ J. J. Song, ${ }^{41}$ W. M. Song, ${ }^{27,1}$ Y. X. Song, ${ }^{38, k}$ S. Sosio, ${ }^{66 a, 66 c}$ S. Spataro, ${ }^{66 a, 66 c}$ K. X. Su, ${ }^{68}$ P. P. Su, ${ }^{46}$ F. F. Sui, ${ }^{41}$ G. X. Sun, ${ }^{1}$ H. K. Sun, ${ }^{1}$ J. F. Sun, ${ }^{16}$ L. Sun, ${ }^{68}$ S. S. Sun, ${ }^{1,54}$ T. Sun, ${ }^{1,54}$ W. Y. Sun, ${ }^{34}$ W. Y. Sun,${ }^{27}$ X. Sun, ${ }^{20,1}$ Y. J. Sun, ${ }^{63,49}$ Y. K. Sun, ${ }^{63,49}$ Y.Z. Sun, ${ }^{1}$ Z. T. Sun, ${ }^{1}$ Y. H. Tan,${ }^{68}$ Y. X. Tan ${ }^{63,49}$ C. J. Tang, ${ }^{45}$ G. Y. Tang, ${ }^{1}$ J. Tang, ${ }^{50}$ J. X. Teng, ${ }^{63,49}$ V. Thoren, ${ }^{67}$ W. H. Tian, ${ }^{43}$ Y. T. Tian, ${ }^{25}$ I. Uman, ${ }^{53 b}$ B. Wang, ${ }^{1}$ C. W. Wang, ${ }^{35}$ D. Y. Wang, ${ }^{38, k}$ H. J. Wang, ${ }^{31, n, o}$ H. P. Wang, ${ }^{1,54}$ K. Wang, ${ }^{1,49}$ L. L. Wang, ${ }^{1}$ M. Wang, ${ }^{41}$ M. Z. Wang, ${ }^{38, k}$ Meng Wang, ${ }^{1,54}$ W. Wang, ${ }^{50}$ W. H. Wang, ${ }^{68}$ W. P. Wang, ${ }^{63,49}$ X. Wang, ${ }^{38, \mathrm{k}}$ X. F. Wang, ${ }^{31, \mathrm{n}, \mathrm{o}}$ X. L. Wang, ${ }^{9, \mathrm{~h}}$ Y. Wang, ${ }^{50}$ Y. Wang, ${ }^{6,49}$ Y. D. Wang, ${ }^{37}$ Y. F. Wang, ${ }^{1,49,54}$ Y. Q. Wang, ${ }^{1}$ Y. Y. Wang, ${ }^{31, n, 0}$ Z. Wang, ${ }^{1,49}$ Z. Y. Wang, ${ }^{1}$ Ziyi Wang, ${ }^{54}$ Zongyuan Wang, ${ }^{1,54}$ D. H. Wei, ${ }^{12}$ F. Weidner ${ }^{60}$ S. P. Wen, ${ }^{1}$ D. J. White, ${ }^{58}$ U. Wiedner, ${ }^{4}$ G. Wilkinson, ${ }^{61}$ M. Wolke, ${ }^{67}$ L. Wollenberg, ${ }^{4}$ J. F. Wu, ${ }^{1,54}$ L. H. Wu, ${ }^{1}$ L. J. Wu, ${ }^{1,54}$ X. Wu, ${ }^{9,}$ Z. Wu, ${ }^{1,49}$ L. Xia,${ }^{63,49}$ H. Xiao, ${ }^{9, h}$ S. Y. Xiao, ${ }^{1}$ Z. J. Xiao, ${ }^{34}$ X. H. Xie, ${ }^{38, k}$ Y. G. Xie, ${ }^{1,49}$ Y. H. Xie ${ }^{6}$ T. Y. Xing, ${ }^{1,54}$ G. F. Xu ${ }^{1}$ Q. J. Xu, ${ }^{14}$ W. Xu, ${ }^{1,54}$ X. P. Xu, ${ }^{46}$ Y. C. Xu ${ }^{54}$ F. Yan, ${ }^{9, h}$ L. Yan, ${ }^{9, h}$ W. B. Yan, ${ }^{63,49}$ W. C. Yan, ${ }^{71}$ Xu Yan, ${ }^{46}$ H. J. Yang, ${ }^{42, g}$ H. X. Yang, ${ }^{1}$ L. Yang, ${ }^{43}$ S. L. Yang, ${ }_{1,54}^{54}$ Y. Xang, ${ }_{1}^{12}$ Yifan Yang, ${ }^{1,54}$ Zhi Yang,${ }^{25}$ M. Ye,${ }^{1,49}$ M. H. Ye, ${ }^{7}$ J. H. Yin, ${ }^{1}$ Z. Y. You, ${ }^{50}$ B.X. Yu, ${ }^{1,4,54}$ C.X. Yu, ${ }^{36}$ G. Yu, ${ }^{1,54}$ J. S. Yu, ${ }^{20,1}$ T. Yu, ${ }^{64}$ C. Z. Yuan, ${ }^{1,54}$ L. Yuan, ${ }^{2}$ X. Q. Yuan, ${ }^{38, k}$ Y. Yuan, ${ }^{1} Z$ Z. Y. Yuan, ${ }^{50}$ C. X. Yue, ${ }^{32}$ A. Yuncu, ${ }^{53 a, a}$ A. A. Zafar, ${ }^{65}$ Zeng, ${ }^{6}$ Y. Zeng, ${ }^{20,1}$ A. Q. Zhang, ${ }^{1}$ B. X. Zhang, ${ }^{1}$ Guangyi Zhang, ${ }^{16}$ H. Zhang, ${ }^{63}$ 
H. H. Zhang, ${ }^{50}$ H. H. Zhang, ${ }^{27}$ H. Y. Zhang, ${ }^{1,49}$ J. J. Zhang, ${ }^{43}$ J. L. Zhang, ${ }^{69}$ J. Q. Zhang, ${ }^{34}$ J. W. Zhang, ${ }^{1,49,54}$ J. Y. Zhang, ${ }^{1}$ J. Z. Zhang, ${ }^{1,54}$ Jianyu Zhang, ${ }^{1,54}$ Jiawei Zhang, ${ }_{1,54}$ L. M. Zhang, ${ }^{52}$ L. Q. Zhang, ${ }^{50}$ Lei Zhang, ${ }^{35}$ S. Zhang, ${ }^{50}$ S. F. Zhang, ${ }^{35}$ Shulei Zhang, ${ }^{20,1}$ X. D. Zhang, ${ }^{37}$ X. Y. Zhang, ${ }^{41}$ Y. Zhang, ${ }^{61}$ Y. H. Zhang, ${ }^{1,49}$ Y. T. Zhang, ${ }^{63,49}$ Yan Zhang, ${ }^{63,49}$ Yao Zhang, Yi Zhang, ${ }^{9,4}$ Z. H. Zhang, ${ }^{6}$ Z. Y. Zhang, ${ }^{68}$ G. Zhao, ${ }^{1}$ J. Zhao, ${ }^{32}$ J. Y. Zhao, ${ }^{1,54}$ J. Z. Zhao, ${ }^{1,49}$ Lei Zhao, ${ }^{63,49}$ Ling Zhao, ${ }^{1}$ M. G. Zhao, ${ }^{36}$ Q. Zhao, ${ }^{1}$ S. J. Zhao, ${ }^{71}$ Y. B. Zhao, ${ }^{1,49}$ Y. X. Zhao, ${ }^{25}$ Z. G. Zhao, ${ }^{63,49}$ A. Zhemchugov, ${ }^{29, b}$ B. Zheng, ${ }^{64}$ J. P. Zheng, ${ }^{1,49}$ Y. Zheng, ${ }^{38, k}$ Y. H. Zheng, ${ }^{54}$ B. Zhong, ${ }^{34}$ C. Zhong, ${ }^{64}$ L. P. Zhou, ${ }^{1,54}$ Q. Zhou, ${ }^{1,54}$ X. Zhou, ${ }^{68}$ X. K. Zhou, ${ }^{54}$ X. R. Zhou, ${ }^{63,49}$ X. Y. Zhou, ${ }^{32}$ A. N. Zhu, ${ }^{1,54}$ J. Zhu, ${ }^{36}$ K. Zhu, ${ }^{1}$ K. J. Zhu, ${ }^{1,49,54}$ S. H. Zhu, ${ }^{62}$ T. J. Zhu, ${ }^{69}$ W. J. Zhu, ${ }^{9, h}$ W. J. Zhu, ${ }^{36}$ Y. C. Zhu, ${ }^{63,49}$ Z. A. Zhu, ${ }^{1,54}$ B. S. Zou, ${ }^{1}$ and J. H. Zou ${ }^{1}$

\title{
(BESIII Collaboration)
}

\author{
${ }^{1}$ Institute of High Energy Physics, Beijing 100049, People's Republic of China \\ ${ }^{2}$ Beihang University, Beijing 100191, People's Republic of China \\ ${ }^{3}$ Beijing Institute of Petrochemical Technology, Beijing 102617, People's Republic of China \\ ${ }^{4}$ Bochum Ruhr-University, D-44780 Bochum, Germany \\ ${ }^{5}$ Carnegie Mellon University, Pittsburgh, Pennsylvania 15213, USA \\ ${ }^{6}$ Central China Normal University, Wuhan 430079, People's Republic of China \\ ${ }^{7}$ China Center of Advanced Science and Technology, Beijing 100190, People's Republic of China \\ ${ }^{8}$ COMSATS University Islamabad, Lahore Campus, Defence Road, \\ Off Raiwind Road, 54000 Lahore, Pakistan \\ ${ }^{9}$ Fudan University, Shanghai 200443, People's Republic of China \\ ${ }^{10}$ G.I. Budker Institute of Nuclear Physics SB RAS (BINP), Novosibirsk 630090, Russia \\ ${ }^{11}$ GSI Helmholtzcentre for Heavy Ion Research GmbH, D-64291 Darmstadt, Germany \\ ${ }^{12}$ Guangxi Normal University, Guilin 541004, People's Republic of China \\ ${ }^{13}$ Guangxi University, Nanning 530004, People's Republic of China \\ ${ }^{14}$ Hangzhou Normal University, Hangzhou 310036, People's Republic of China \\ ${ }^{15}$ Helmholtz Institute Mainz, Staudinger Weg 18, D-55099 Mainz, Germany \\ ${ }^{16}$ Henan Normal University, Xinxiang 453007, People's Republic of China \\ ${ }^{17}$ Henan University of Science and Technology, Luoyang 471003, People's Republic of China \\ ${ }^{18}$ Huangshan College, Huangshan 245000, People's Republic of China \\ ${ }^{19}$ Hunan Normal University, Changsha 410081, People's Republic of China \\ ${ }^{20}$ Hunan University, Changsha 410082, People's Republic of China \\ ${ }^{21}$ Indian Institute of Technology Madras, Chennai 600036, India \\ ${ }^{22}$ Indiana University, Bloomington, Indiana 47405, USA \\ ${ }^{23 a}$ INFN Laboratori Nazionali di Frascati, I-00044 Frascati, Italy \\ ${ }^{23 \mathrm{~b}}$ INFN Sezione di Perugia, I-06100 Perugia, Italy \\ ${ }^{23 c}$ University of Perugia, I-06100 Perugia, Italy \\ ${ }^{24 a}$ INFN Sezione di Ferrara, I-44122 Ferrara, Italy \\ ${ }^{24 \mathrm{~b}}$ University of Ferrara, I-44122 Ferrara, Italy \\ ${ }^{25}$ Institute of Modern Physics, Lanzhou 730000, People's Republic of China \\ ${ }^{26}$ Institute of Physics and Technology, Peace Avenue 54B, Ulaanbaatar 13330, Mongolia \\ ${ }^{27}$ Jilin University, Changchun 130012, People's Republic of China \\ ${ }^{28}$ Johannes Gutenberg University of Mainz, Johann-Joachim-Becher-Weg 45, D-55099 Mainz, Germany \\ ${ }^{29}$ Joint Institute for Nuclear Research, 141980 Dubna, Moscow region, Russia \\ ${ }^{30}$ Justus-Liebig-Universitaet Giessen, II. Physikalisches Institut, \\ Heinrich-Buff-Ring 16, D-35392 Giessen, Germany \\ ${ }^{31}$ Lanzhou University, Lanzhou 730000, People's Republic of China \\ ${ }^{32}$ Liaoning Normal University, Dalian 116029, People's Republic of China \\ ${ }^{33}$ Liaoning University, Shenyang 110036, People's Republic of China \\ ${ }^{34}$ Nanjing Normal University, Nanjing 210023, People's Republic of China \\ ${ }^{35}$ Nanjing University, Nanjing 210093, People's Republic of China \\ ${ }^{36}$ Nankai University, Tianjin 300071, People's Republic of China \\ ${ }^{37}$ North China Electric Power University, Beijing 102206, People's Republic of China \\ ${ }^{38}$ Peking University, Beijing 100871, People's Republic of China \\ ${ }^{39}$ Qufu Normal University, Qufu 273165, People's Republic of China \\ ${ }^{40}$ Shandong Normal University, Jinan 250014, People's Republic of China \\ ${ }^{41}$ Shandong University, Jinan 250100, People's Republic of China \\ ${ }^{42}$ Shanghai Jiao Tong University, Shanghai 200240, People's Republic of China \\ ${ }^{43}$ Shanxi Normal University, Linfen 041004, People's Republic of China
}




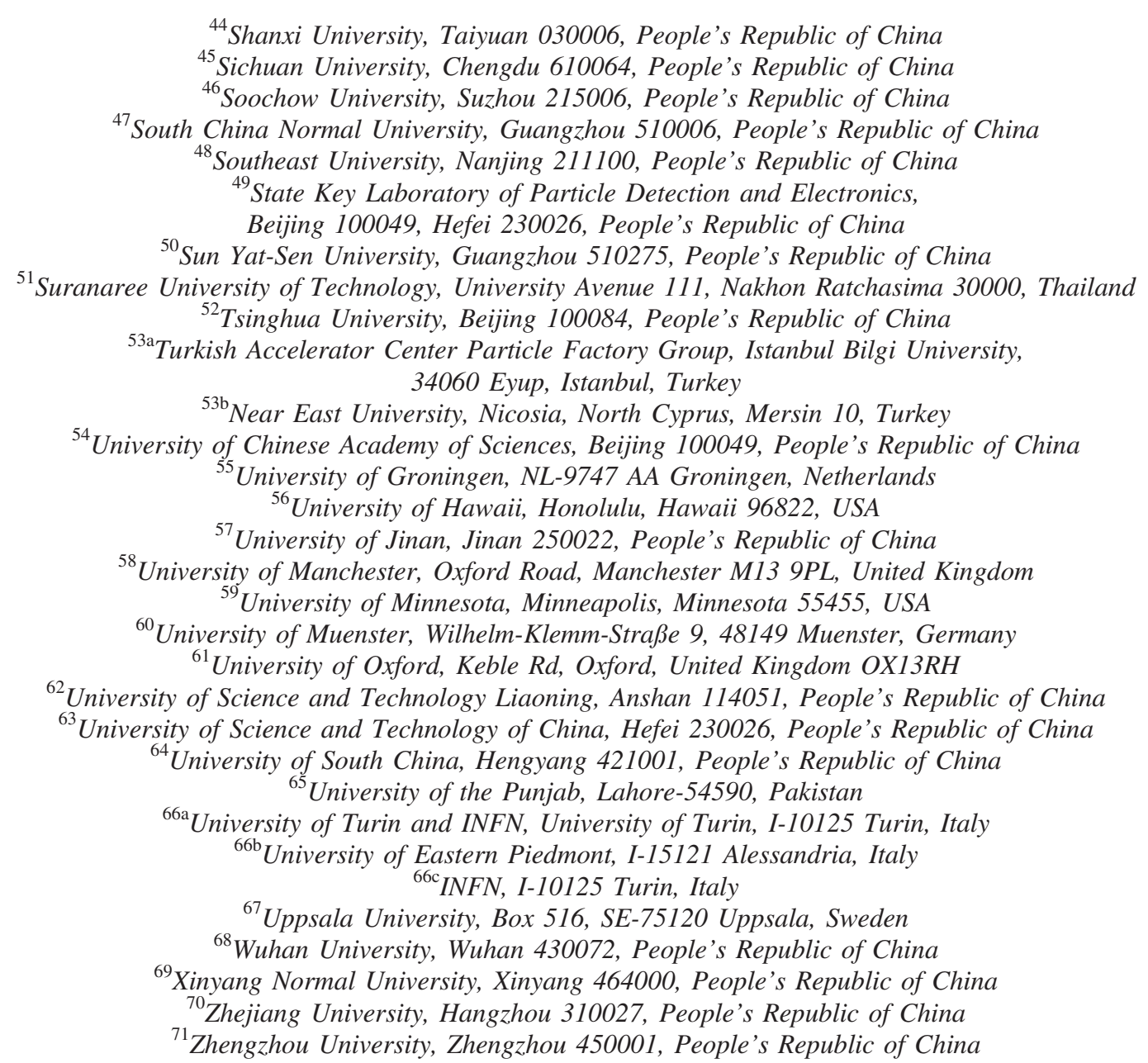

(Received 31 October 2020; revised 16 February 2021; accepted 12 April 2021; published 19 May 2021)

We report on a comparison of two possible $\Lambda_{c}^{+}$spin hypotheses, $J=\frac{1}{2}$ and $\frac{3}{2}$, via the process $e^{+} e^{-} \rightarrow \Lambda_{c}^{+} \bar{\Lambda}_{c}^{-}$, using the angular distributions of $\Lambda_{c}^{+}$decays into $p K_{S}^{0}, \Lambda \pi^{+}, \Sigma^{0} \pi^{+}$, and $\Sigma^{+} \pi^{0}$. The data

${ }^{\mathrm{a} A}$ Also at Bogazici University, 34342 Istanbul, Turkey.

${ }^{\mathrm{b}}$ Also at the Moscow Institute of Physics and Technology, Moscow 141700, Russia.

${ }^{\mathrm{c}}$ Also at the Novosibirsk State University, Novosibirsk 630090, Russia.

"Also at the NRC "Kurchatov Institute", PNPI, 188300 Gatchina, Russia.

${ }^{\mathrm{e}}$ Also at Istanbul Arel University, 34295 Istanbul, Turkey.

${ }^{\mathrm{f}}$ Also at Goethe University Frankfurt, 60323 Frankfurt am Main, Germany.

${ }^{g}$ Also at Key Laboratory for Particle Physics, Astrophysics and Cosmology, Ministry of Education; Shanghai Key Laboratory for Particle Physics and Cosmology; Institute of Nuclear and Particle Physics, Shanghai 200240, People's Republic of China.

${ }^{\mathrm{h}}$ Also at Key Laboratory of Nuclear Physics and Ion-beam Application (MOE) and Institute of Modern Physics, Fudan University, Shanghai 200443, People's Republic of China.

${ }^{i}$ Also at Harvard University, Department of Physics, Cambridge, Massachusets 02138, USA.

${ }^{j}$ Present address: Institute of Physics and Technology, Peace Avenue 54B, Ulaanbaatar 13330, Mongolia.

${ }^{k}$ Also at State Key Laboratory of Nuclear Physics and Technology, Peking University, Beijing 100871, People's Republic of China. ${ }^{1}$ School of Physics and Electronics, Hunan University, Changsha 410082, China.

${ }^{\mathrm{m}}$ Also at Guangdong Provincial Key Laboratory of Nuclear Science, Institute of Quantum Matter, South China Normal University, Guangzhou 510006, China.

${ }^{\mathrm{n}}$ Frontier Science Center for Rare Isotopes, Lanzhou University, Lanzhou 730000, People's Republic of China.

${ }^{\circ}$ Lanzhou Center for Theoretical Physics, Lanzhou University, Lanzhou 730000, People's Republic of China.

${ }^{\mathrm{p}}$ Frontiers Science Center for Rare Isotopes, Lanzhou University, Lanzhou 730000, People's Republic of China.

Published by the American Physical Society under the terms of the Creative Commons Attribution 4.0 International license. Further distribution of this work must maintain attribution to the author(s) and the published article's title, journal citation, and DOI. Funded by SCOAP. 
were recorded at $\sqrt{s}=4.6 \mathrm{GeV}$ with the BESIII detector and correspond to an integrated luminosity of $587 \mathrm{pb}^{-1}$. The $\Lambda_{c}^{+}$spin is determined to be $J=\frac{1}{2}$, with this value favored over the $\frac{3}{2}$ hypothesis with a significance corresponding to more than 6 Gaussian standard deviations.

DOI: 10.1103/PhysRevD.103.L091101

Since the discovery of the $\Lambda_{c}^{+}$particle more than 30 years ago [1], many other charmed baryons have been found and studied by experiments [2]. However, the $\Lambda_{c}^{+}$spin quantum number has not been determined conclusively until now. Unlike stable particles, whose spin can be measured with a dedicated detector, e.g., Stern-Gerlach setup, the spin of short-lived $\Lambda_{c}^{+}$can be only studied via its decays. Although the spin quantum number can be inferred from the phenomenological Regge trajectory [3-8], the establishment of the $\Lambda_{c}^{+}$spin needs a direct experimental measurement, making use of information on the angular distribution for the decayed particles. Thus a large size and clean data events are need in the analysis. The only previous investigation of this property was performed by the NA32 fixedtarget experiment [9]. The charmed baryon $\Lambda_{c}^{+}$was produced in the process $\pi^{-} \mathrm{Cu} \rightarrow \Lambda_{c}^{+} \bar{D} X$, where $X$ indicates the other particles produced from the interaction, and the decay $\Lambda_{c}^{+} \rightarrow p K^{-} \pi^{+}$was used to reconstruct the charmed baryon with 160 selected candidate events. The result was compatible with a spin-1/2 assignment, but was not conclusive due to the small sample size.

Currently, the spin of the $\Lambda_{c}^{+}$is inferred to be $\frac{1}{2}$ from the naive quark model [10], in which charmed baryons are built from $u d c$ quarks, and $\Lambda_{c}^{+}$is classified into the mixedsymmetric 20 multiplet with spin-1/2 assignment. Theoretically, the $\Lambda_{c}^{+}$system is suggested as a unique and excellent laboratory to study heavy quark symmetry and chiral symmetry of the $u, d$ light quarks [4-6]. A large number of theoretical predictions on the $\Lambda_{c}^{+}$properties and decays are made based on the spin-1/2 assumption [4-6]. Although the quark model works well for the ground states [2], experimental confirmation of the $\Lambda_{c}^{+}$spin is essential for testing the quark model spin assignment and theoretical predictions. Knowledge of the $\Lambda_{c}^{+}$spin is also important for measurement of its intrinsic properties, such as its anomalous magnetic moment [11], magnetic dipole moments [12] and electromagnetic dipole moments $[13,14]$. Moreover, its decays can be used as a spin polarimeter [15] to determine the $c$-quark polarization at the Large Hadron Collider. Furthermore the $\Lambda_{c}^{+}$spin and polarization are intimately related to the understanding of other charmed baryon properties, e.g., the newly observed $\Xi_{c c}^{++}$[16], which decays into final states with $\Lambda_{c}^{+}$.

In this paper, an analysis of the $\Lambda_{c}^{+}$spin is performed via the process $e^{+} e^{-} \rightarrow \Lambda_{c}^{+} \bar{\Lambda}_{c}^{-}$at the center-of-mass (CM) energy $\sqrt{s}=4.6 \mathrm{GeV}$. The data accumulated with the BESIII [17] detector corresponds to an integrated luminosity of $587 \mathrm{pb}^{-1}$. We test the spin- $1 / 2$ and $3 / 2$ hypotheses based on the angular distributions of the $\Lambda_{c}^{+}$ decays into $p K_{S}^{0}, \Lambda \pi^{+}, \Sigma^{0} \pi^{+}$and $\Sigma^{+} \pi^{0}$. The decays are studied by the single-tag method, i.e., either the $\Lambda_{c}^{+}$or $\bar{\Lambda}_{c}^{-}$ from $e^{+} e^{-} \rightarrow \Lambda_{c}^{+} \bar{\Lambda}_{c}^{-}$is reconstructed while the presence of its recoiled $\Lambda_{c}^{+}$or $\bar{\Lambda}_{c}^{-}$is inferred from kinematics. Throughout the paper, the charged-conjugation modes are always implied, unless explicitly stated.

The helicity formalism $[18,19]$ is applied in order to examine the implications of the $\Lambda_{c}^{+}$spin hypotheses for the joint angular distribution of the charmed baryon and its daughter particles. Figure 1 shows the helicity frame for the $e^{+} e^{-} \rightarrow \Lambda_{c}^{+} \bar{\Lambda}_{c}^{-}$process. The helicity angle, $\theta_{0}$, is defined as the polar angle of the $\Lambda_{c}^{+}$in the $e^{+} e^{-} \mathrm{CM}$ system, with the $z$ axis pointing along the positron beam direction. For the $\Lambda_{c}^{+}$decay into a spin $-\frac{1}{2}$ baryon $(B)$ and a pseudoscalar meson $(P)$, the $z^{\prime}$ axis is defined along the direction of the $\Lambda_{c}^{+}$, and $y^{\prime}$ axis along $\hat{z} \times \hat{z}^{\prime}$, and the $x^{\prime}$ axis is determined by $\hat{y}^{\prime} \times \hat{z}^{\prime}$. The helicity angle $\phi_{1}$ is defined as the angle between the $\Lambda_{c}^{+}$production and decay planes and the helicity angle $\theta_{1}$ is the angle between the $B$ momentum in the $\Lambda_{c}^{+}$rest frame and the $z^{\prime}$ axis. The helicity angles for the subsequential baryon $B$ decays, $\left(\theta_{i}, \phi_{i}\right)$ with $i>2$, can be defined following the same procedure.

The two $\Lambda_{c}^{+}$spin hypotheses, $J=\frac{1}{2}$ or $\frac{3}{2}$, are tested using $\mathcal{W}^{J}$, which is the trace of the product of three matrices describing the joint angular distribution of the sequential decays:

$$
\mathcal{W}^{J}=\operatorname{Tr}\left[\rho_{J} \cdot \mathcal{T}_{\mathcal{J}} \cdot \mathcal{T}_{\mathcal{B}}\right]
$$

Here $\rho_{J}$ is the spin density matrix for a $\Lambda_{c}^{+}$baryon produced in the process $e^{+} e^{-} \rightarrow \Lambda_{c}^{+} \bar{\Lambda}_{c}^{-}, \mathcal{T}_{J}$ is a matrix describing the $\Lambda_{c}^{+}$decay to a baryon $B$ and a pseudoscalar meson $P$, and the baryon $B$ sequentially decaying to the final states is described with a matrix $\mathcal{T}_{\mathcal{B}}$. The full formulas can be found in Refs [20-22]. As an example, the $\Lambda_{c}^{+} \rightarrow p K_{S}^{0}$, decay

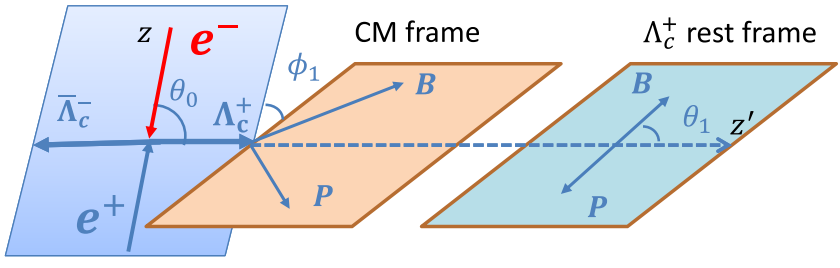

FIG. 1. Definition of the helicity frame for $e^{+} e^{-} \rightarrow \Lambda_{c}^{+} \bar{\Lambda}_{c}^{-}$, $\Lambda_{c}^{+} \rightarrow B P$, where $B$ and $P$ denote a spin- $\frac{1}{2}$ baryon and a meson, respectively. 
matrix, $\mathcal{T}_{\mathcal{B}}$, reduces to the identity matrix, and the joint angular distribution is given by

$$
\begin{aligned}
\mathcal{W}^{J=\frac{1}{2}}\left(\theta_{0}, \theta_{1}, \phi_{1}\right) & \propto 1+\alpha \cos ^{2} \theta_{0}+\mathcal{P}_{\mathcal{T}} \sin \theta_{1} \sin \phi_{1}, \\
\text { with } \quad \mathcal{P}_{\mathcal{T}} & =\alpha_{\left[p K_{S}^{0}\right]} \sqrt{1-\alpha^{2}} \cos \theta_{0} \sin \theta_{0} \sin \xi
\end{aligned}
$$

where $\alpha$ is the angular-distribution parameter of the $\Lambda_{c}^{+}$, $\alpha_{\left[p K_{S}^{0}\right]}$ the asymmetry parameter for the $\Lambda_{c}^{+} \rightarrow p K_{S}^{0}$ weak decay and $\xi$ the relative phase between the two independent helicity amplitudes of the produced $\Lambda_{c}^{+}$.

The joint angular distribution derived for the spin $-\frac{3}{2}$ hypothesis for $\Lambda_{c}^{+} \rightarrow p K_{S}^{0}$ is [21]

$$
\begin{aligned}
\mathcal{W}^{J=\frac{3}{2}}\left(\theta_{0}, \theta_{1}, \phi_{1}\right) \propto & 40 r_{0}^{0}-10 \sqrt{3} r_{0}^{2}\left(3 \cos 2 \theta_{1}+1\right) \\
& -60\left[r_{1}^{2} \sin 2 \theta_{1} \cos \phi_{1}+r_{2}^{2} \sin ^{2} \theta_{1} \cos 2 \phi_{1}\right] \\
& +\sin \theta_{1} \alpha_{\left[p K_{S}^{0}\right]}\left[8 \sqrt{15} r_{-1}^{1} \sin \phi_{1}\right. \\
& +90 r_{-2}^{3} \sin 2 \theta_{1} \sin 2 \phi_{1} \\
& \left.-9 \sqrt{10} r_{-1}^{3}\left(5 \cos 2 \theta_{1}+3\right) \sin \phi_{1}\right],
\end{aligned}
$$

where the real multipole parameters, $r_{M}^{L}$, are defined in terms of the helicity amplitudes for charmed baryon pair production [21].

The BESIII detector is an approximately cylindrically symmetric detector with $93 \%$ coverage of the solid angle around the $e^{+} e^{-}$interaction point (IP). The components of the apparatus are a helium-based main drift chamber (MDC), a plastic time-of-flight (TOF) system, a 6240-cell $\mathrm{CsI}(\mathrm{Tl})$ crystal electromagnetic calorimeter (EMC), a superconducting solenoid providing a $1.0 \mathrm{~T}$ magnetic field aligned with the beam axis, and a muon counter with resistive plate chambers as the active element. The momentum resolution for charged tracks in the MDC is $0.5 \%$ for a transverse momentum of $1 \mathrm{GeV} / c$. The photon energy resolution in the EMC is $2.5 \%$ in the barrel region and $5.0 \%$ in the end-cap region for $1 \mathrm{GeV}$ photons. The combined information of the energy deposit in the MDC and the flight time measured by the TOF is used for particle identification (PID) of charged tracks. More details about the design and performance of the BESIII detector are given in Ref. [23].

We use a large Monte Carlo (MC) simulated sample of $e^{+} e^{-}$annihilations to understand background and to estimate the detection efficiencies. The event generation is performed by the KKMC generator [24], taking the beamenergy spread and initial-state radiation (ISR) into account. Inclusive MC samples, consisting of generic $\Lambda_{c}^{+} \bar{\Lambda}_{c}^{-}$events, $D_{(s)}^{*} \bar{D}_{(s)}^{*}+X$ production [25], ISR production of lowerlying charmonium(-like) $\psi$ states as well as continuum processes $e^{+} e^{-} \rightarrow q \bar{q}(q=u, d, s)$ are generated for a complete description of the background. The decays are generated using EvtGen [26] with the decay fractions from
Ref. [2] as input. The propagation through the detector and material interactions are simulated by using GEANT4 [27].

The $\Lambda_{c}^{+}$candidates are reconstructed from the $p K_{S}^{0}, \Lambda \pi^{+}$, $\Sigma^{+} \pi^{0}$, and $\Sigma^{0} \pi^{+}$final states as done in Refs. [22,28]. The intermediate states, $K_{S}^{0}, \Lambda, \Sigma^{+}, \Sigma^{0}$ and $\pi^{0}$, are reconstructed from the $\pi^{+} \pi^{-}, p \pi^{-}, p \pi^{0}, \gamma \Lambda$ and $\gamma \gamma$ decays, respectively.

Charged tracks are required to originate from the interaction region, defined by a cylinder with a radius of $1 \mathrm{~cm}$ and a distance from the IP along the beam direction of $\pm 10 \mathrm{~cm}$, except for those charged tracks coming from $\Lambda$ and $K_{S}^{0}$ decays. The polar angle $\theta$ of each track with respect to the beam direction is required to fulfill $|\cos \theta|<0.93$. Protons are identified by requiring the PID likelihood $\mathcal{L}$ to satisfy $\mathcal{L}(p)>\mathcal{L}(K)$ and $\mathcal{L}(p)>\mathcal{L}(\pi)$, while charged pions are identified using $\mathcal{L}(\pi)>\mathcal{L}(K)$, except for those from $\Lambda$ and $K_{S}^{0}$ decays.

Clusters in the EMC with no associated charged tracks are identified as photon candidates if the energy deposit in the barrel region $(|\cos \theta|<0.80)$ is larger than $25 \mathrm{MeV}$, or if in the endcap region $(0.86<|\cos \theta|<0.92)$ it is larger than $50 \mathrm{MeV}$. To suppress background from electronic noise and coincidental EMC showers, the difference between the event start time and EMC signal is required to be smaller than $700 \mathrm{~ns}$. The $\pi^{0}$ candidates are reconstructed from photon pairs with an invariant mass, $M(\gamma \gamma)$, which satisfies $115<M(\gamma \gamma)<150 \mathrm{MeV} / c^{2}$. To improve the momentum resolution, a mass-constrained fit to the $\pi^{0}$ nominal mass is applied to the photon pairs and the resulting $\pi^{0}$ energy and momentum is used for the further analysis.

The $\Lambda\left(K_{S}^{0}\right)$ candidates are formed by combining the final states $p \pi^{-}\left(\pi^{+} \pi^{-}\right)$with a displacement less than $20 \mathrm{~cm}$ from the IP along the electron beam direction. The $\chi^{2}$ of the vertex fit is required to be smaller than 100 and the distance from the IP must be larger than twice the vertex resolution. The momenta of the daughter particles obtained from the fit are used in the further analysis. The charged pions associated with the $\Lambda$ and $K_{S}^{0}$ candidates are not subjected to any PID requirement, while proton PID is applied in order to improve the signal significance. To select $K_{S}^{0}, \Lambda, \Sigma^{0}$, and $\Sigma^{+}$candidates, we require $487<M\left(\pi^{+} \pi^{-}\right)<511 \mathrm{MeV} / c^{2}, 1111<M\left(p \pi^{-}\right)<$ $1121 \mathrm{MeV} / c^{2}, \quad 1179<M(\Lambda \gamma)<1203 \mathrm{MeV} / c^{2}, \quad$ and $1176<M\left(p \pi^{0}\right)<1200 \mathrm{MeV} / c^{2}$, respectively. These requirements correspond to windows of approximately \pm 3 standard deviations around the nominal masses. In order to remove $p K_{S}^{0}, K_{S}^{0} \rightarrow \pi^{0} \pi^{0}$ background in the $\Sigma^{+} \pi^{0}$ sample, the mass of the $\pi^{0} \pi^{0}$ pair is required to lie outside the range $(400,550) \mathrm{MeV} / c^{2}$.

The $\Lambda_{c}^{+}$candidates in each decay mode are selected by requiring the beam-constrained mass $M_{\mathrm{BC}} \equiv \sqrt{E_{\text {beam }}^{2}-p_{\Lambda_{c}^{+}}^{2}}$ to be within the range $(2.278,2.294) \mathrm{GeV} / c^{2}$, where $E_{\text {beam }}$ is the beam energy and $p_{\Lambda_{c}^{+}}$is the measured $\Lambda_{c}^{+}$momentum 


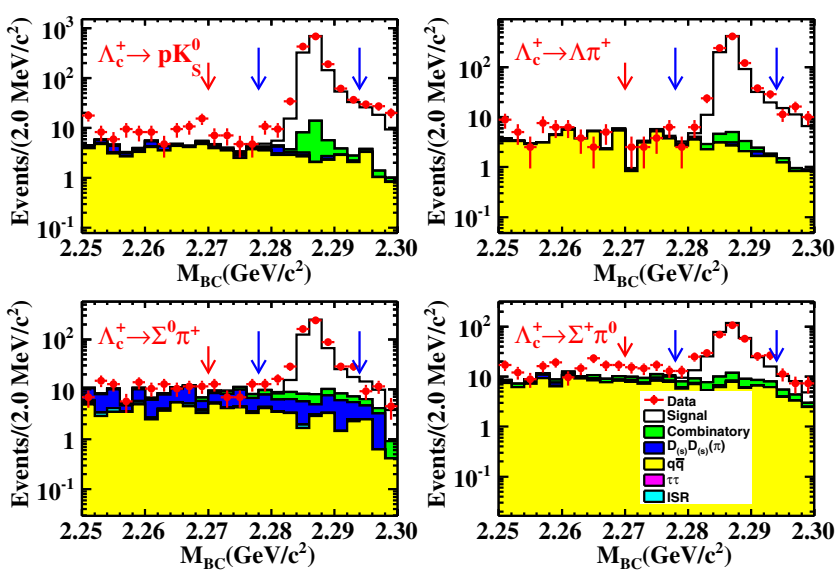

FIG. 2. The $M_{\mathrm{BC}}$ distributions for different decay modes. Points with error bars represent the $M_{\mathrm{BC}}$ distribution for the data, unfilled histograms for signal MC samples and shaded histograms for MC simulated background. The long vertical arrows indicate the $\Lambda_{c}^{+}$mass window, while the sideband region is to the left of the short arrow.

in the CM system of the $e^{+} e^{-}$collision. The numbers of reconstructed $\Lambda_{c}^{+}$candidates are 1227, 696, 614 and 412 for the $p K_{S}^{0}, \Lambda \pi^{+}, \Sigma^{0} \pi^{+}$and $\Sigma^{+} \pi^{0}$ modes, respectively. If multiple candidates are found in a single event, we keep the one with the smallest energy difference $|\Delta E|$, where $\Delta E \equiv$ $E_{\Lambda_{c}^{+}}-E_{\text {beam }}$ and $E_{\Lambda_{c}^{+}}$is the total measured energy of the $\Lambda_{c}^{+}$candidate. To improve the signal purity, $\Delta E$ is required to be smaller than three times the resolution of energy difference distribution. The $M_{\mathrm{BC}}$ distributions of the different $\Lambda_{c}^{+}$decay modes are shown in Fig. 2. The $\Lambda_{c}^{+}$ candidates appear as a peak at the nominal $\Lambda_{c}^{+}$mass whereas the backgrounds, studied in inclusive MC samples, have smooth $M_{\mathrm{BC}}$ distributions that are modeled with an Argus function [29]. The background level in the signal region can be estimated from sidebands, defined by $M_{\mathrm{BC}}$ values within the range $(2.250,2.270) \mathrm{GeV} / c^{2}$. Table I lists the numbers of observed $\left(N^{\mathrm{obs}}\right)$ and normalized background events $\left(N^{\mathrm{bg}}\right)$ in the $\Lambda_{c}^{+}$signal region, where $N^{\text {bg }}$ is estimated from the sideband.

TABLE I. Summary of observed $\left(N^{\text {obs }}\right)$ and normalized background events $\left(N^{\mathrm{bg}}\right)$ in the $\Lambda_{c}^{+}$signal region, where $N^{\mathrm{bg}}$ is estimated from the sideband.

\begin{tabular}{lcc}
\hline \hline Decay & $N^{\text {obs }}$ & $N^{\mathrm{bg}}$ \\
\hline$\Lambda_{c}^{+} \rightarrow p K_{S}^{0}$ & 618 & 25.4 \\
$\bar{\Lambda}_{c}^{-} \rightarrow \bar{p} K_{S}^{0}$ & 609 & 23.6 \\
$\Lambda_{c}^{+} \rightarrow \Lambda \pi^{+}$ & 352 & 10.6 \\
$\bar{\Lambda}_{c}^{-} \rightarrow \bar{\Lambda} \pi^{-}$ & 344 & 11.8 \\
$\Lambda_{c}^{+} \rightarrow \Sigma^{0} \pi^{+}$ & 251 & 26.3 \\
$\bar{\Lambda}_{c}^{-} \rightarrow \bar{\Sigma}^{0} \pi^{-}$ & 279 & 24.2 \\
$\Lambda_{c}^{+} \rightarrow \Sigma^{+} \pi^{0}$ & 192 & 67.4 \\
$\bar{\Lambda}_{c}^{-} \rightarrow \bar{\Sigma}^{+} \pi^{0}$ & 184 & 52.4 \\
\hline \hline
\end{tabular}

The $\Lambda_{c}^{+}$spin- $J$ hypotheses are tested using a likelihood function, which is defined for a given process $i$ as

$$
\mathcal{L}_{i}^{J}\left(N^{i}\right)=\prod_{k=1}^{N^{i}} \frac{1}{\mathcal{C}^{i}} \mathcal{W}^{J}\left(\theta_{0}^{k}, \theta_{1}^{k}, \phi_{1}^{k}, \ldots, \theta_{n}^{k}, \phi_{n}^{k}\right)
$$

where $N^{i}$ is the number of events of $i$ th decay mode defined in Table I, $\left(\theta_{0}^{k}, \theta_{1}^{k}, \phi_{1}^{k}, \ldots, \theta_{n}^{k}, \phi_{n}^{k}\right)$ are the helicity angles for the $k$-th event with $n$-step $\Lambda_{c}^{+}$decays, and $\mathcal{C}^{i}=$ $\int \mathcal{W}^{J}\left(\theta_{0}, \theta_{1}, \phi_{1}, \ldots, \theta_{n}^{k}, \phi_{n}^{k}\right) \mathrm{d} \cos \theta_{0} \prod_{m=1}^{m=n} \mathrm{~d} \cos \theta_{m} \mathrm{~d} \phi_{m}$ is a normalization factor, calculated using a large phase-space MC sample.

The physics parameters are obtained by applying a simultaneous fit to the joint angular distribution of the selected events in the signal region. The background contributions are subtracted from the log-likelihood values using the weighted sideband events. The net log-likelihood for a given process $i$ is defined by

$$
\ln \mathcal{L}_{i}^{J}=\ln \mathcal{L}_{i}^{J}\left(N_{s}^{i}\right)-\omega^{\mathrm{bg}} \ln \mathcal{L}_{i}^{J}\left(N_{b}^{i}\right),
$$

where $N_{s}^{i}\left(N_{b}^{i}\right)$ is the number of selected data (background) events. The background weight, $\omega^{\mathrm{bg}}$, is the ratio between the number of background events in the signal region, and the number of sideband events. In estimating the background weight, its line shape in the fit is taken as an Argus function.

The MINUIT [30] package is used to minimize the objective function, $S=-\sum_{i} \ln \mathcal{L}_{i}^{J}$, in the simultaneous fit to the $p K_{S}^{0}, \Lambda \pi^{+}, \Sigma^{0} \pi^{+}$, and $\Sigma^{+} \pi^{0}$ decay modes. The decay asymmetry parameters for the spin $-\frac{1}{2}$ hypothesis, e.g., $\alpha_{\left[p K_{S}^{0}\right]}$ in Eq. (2) are constrained to the range $[-1,1]$ in the simultaneous fit. For the spin- $\frac{3}{2}$ hypothesis, the asymmetry parameters in the fit are constrained to be in the physical region [31] for each mode, i.e., $-\frac{1}{3} \leq \alpha_{[B P]} \leq \frac{1}{3}$. The relative phase between the helicity amplitudes are fixed to the expected values near threshold [21], whereas the moduli of the helicity amplitudes in $r_{M}^{L}$ are obtained from the fit. The minimum log-likelihood, given by $-\sum_{i} \ln \mathcal{L}_{i}^{J}$, is determined to be -45.18 for the spin $-\frac{1}{2}$ hypothesis and -21.50 for the spin $-\frac{3}{2}$ hypothesis. Hence, our data favors the spin- $\frac{1}{2}$ hypothesis.

The distribution of estimates of the expectation value $\left\langle\sin 2 \theta_{1} \cos \phi_{1}\right\rangle$ moment, an average observed in each bin, is a useful observable to illustrate the different behaviour expected for the two hypotheses. Figure 3 shows the first moment of the $\left\langle\sin 2 \theta_{1} \cos \phi_{1}\right\rangle$ distribution under the two spin hypotheses for the all-mode-combined events, and the projections of the two fits suggest that the data favor the spin $-\frac{1}{2}$ to the spin- $\frac{3}{2}$ hypothesis.

In order to quantify the discriminating power of the test, we study the likelihood ratio distribution, $t \equiv$ $-2 \ln \left(\mathcal{L}^{J=3 / 2} / \mathcal{L}^{J=1 / 2}\right)$, obtained from a series of $\mathrm{MC}$ 


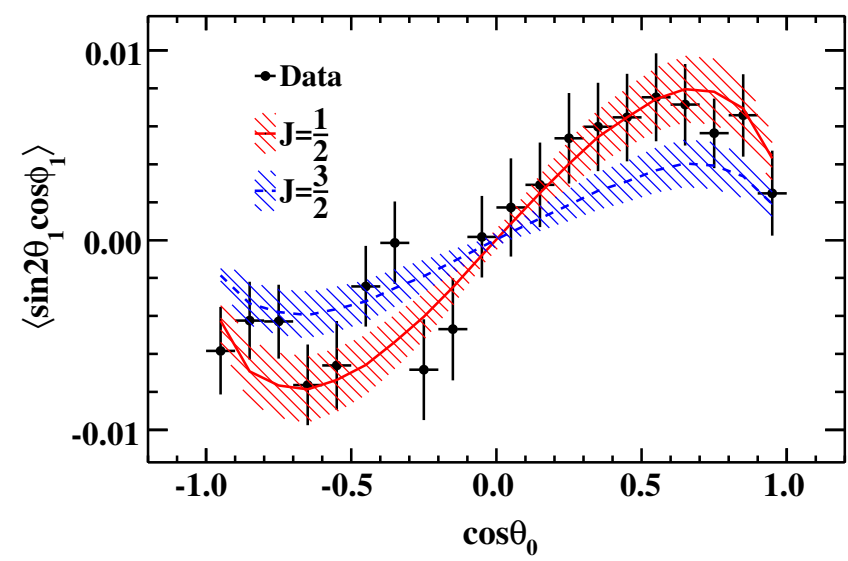

FIG. 3. The moments $\left\langle\sin 2 \theta_{1} \cos \phi_{1}\right\rangle$ as a function of $\cos \theta_{0}$. Points with error bars represent the combined data events from the tagged four $\Lambda_{c}^{+}$decay modes, where the background contribution has been subtracted. The red solid curve shows the fitted result of the spin- $-\frac{1}{2}$ hypothesis, whereas the blue dashed curve shows that of the spin- $\frac{3}{2}$ hypothesis. The bands represent the fit uncertainty due to statistical uncertainties.

simulations, following the method in Ref. [32]. The MC sample for each hypothesis is generated according to its joint angular distribution, propagated through the detector model and subjected to the same event selection criteria as applied to the data events. Each MC subset has the same size as the data sample and is assumed to have the same amount of background. The test statistic $t$ distributions are shown in Fig. 4 for about 20,000 MC simulations. The simulations for the right peak $(t>0)$ are performed under the $J=\frac{1}{2}$ hypothesis, while those in the left peak $(t<0)$ correspond to the $J=\frac{3}{2}$ hypothesis. It is clear that the

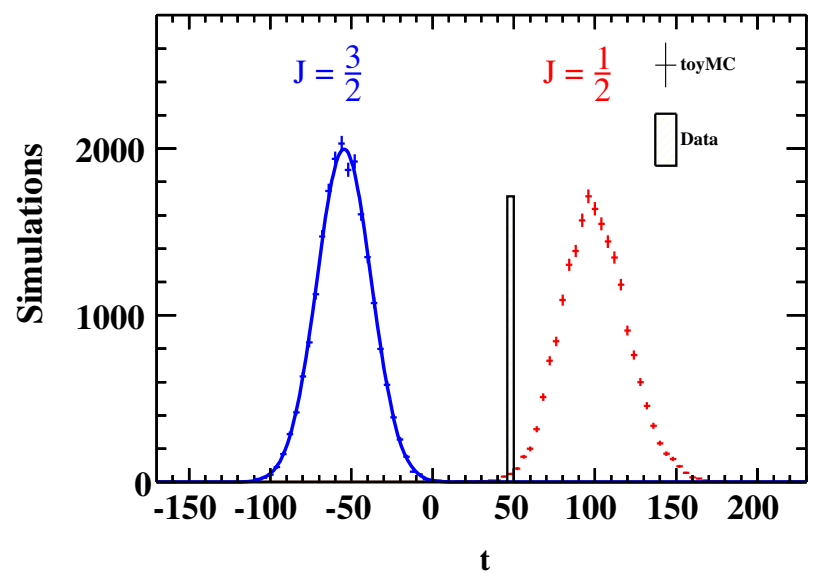

FIG. 4. Distributions of the test statistic $t \equiv$ $-2 \ln \left[\mathcal{L}^{J=3 / 2} / \mathcal{L}^{J=1 / 2}\right]$, for a series of MC simulations performed under the spin- $\frac{1}{2}$ (right peak) and spin- $\frac{3}{2}$ (left peak) hypotheses. The curve is the Gaussian-fitted distribution to the left peak. The $t$ value obtained from experimental data is indicated by the vertical bar. $t$-distributions of the two hypotheses are well separated, and can be discriminated between by setting an acceptance criterion of $t \geq 0$ for $J=\frac{1}{2}$ and $t<0$ for $J=\frac{3}{2}$. Since the $t$-value from the data fulfills $t \geq 0$, as shown in Fig. 4, it is inconsistent with the spin- $\frac{3}{2}$ hypothesis. Hence, our data favor the spin- $\frac{1}{2}$ assignment. The statistical significance for the spin- $\frac{3}{2}$ over spin- $\frac{1}{2}$ hypothesis is estimated approximately with $\left(t_{\text {data }}-\langle t\rangle\right) / \sigma(t)$ [32], where $\langle t\rangle$ and $\sigma(t)$ are the mean and standard deviation for the ensemble of MC simulations under the spin- $-\frac{3}{2}$ hypothesis with $t<0$. We find that the spin- $\frac{3}{2}$ hypothesis can be rejected with a significance of $6.07 \sigma$ in favor of the spin- $-\frac{1}{2}$ hypothesis.

The significance to accept the spin- $-\frac{1}{2}$ hypothesis over the spin- $\frac{3}{2}$ hypothesis can be affected by the systematic sources listed in Table II. We estimate these systematic variations with the same MC method that was used for the likelihood ratio. The results are listed in Table II.

The efficiencies of the tracking and PID for charged tracks and their dependence on transverse momentum and polar angle are measured using a control data sample $J / \psi \rightarrow p \bar{p} \pi^{+} \pi^{-}$decays [33]. The uncertainties associated with the detection efficiency of the radiative photon in $\Sigma^{0} \rightarrow \gamma \Lambda$ decays are assessed with a control sample of $J / \psi \rightarrow \pi^{+} \pi^{-} \pi^{0}, \pi^{0} \rightarrow \gamma \gamma$ decays. The efficiency differences between data and MC simulations are determined to be $0.5 \%$ and $1.5 \%$ in the barrel and endcap region, respectively [34]. The difference between data and MC simulation of the $\pi^{0}$ reconstruction efficiency in the $\Lambda_{c}^{+} \rightarrow \Sigma^{+} \pi^{0}$ decay, and its dependence on momentum, is studied using the processes $\psi(3686) \rightarrow \pi^{0} \pi^{0} J / \psi$ and $e^{+} e^{-} \rightarrow \pi^{0} \omega$ at $\sqrt{s}=3773 \mathrm{MeV}$. The $\Lambda$ reconstruction efficiency is studied as a function of momentum and polar angle in the reaction $\Lambda_{c}^{+} \rightarrow \Lambda+X$ [35]. To take into account the correlations between the different sources of correction and uncertainty, we perform an overall weighting of $\mathrm{MC}$ events in the fit according to these efficiency corrections.

TABLE II. Systematic uncertainties for estimating the significance of spin- $-\frac{1}{2}$ versus the spin- $\frac{3}{2}$ hypothesis determined with the toy MC method.

\begin{tabular}{lc}
\hline \hline Source & Significance \\
\hline Nominal fit & $6.07 \sigma$ \\
Tracks and PID & \\
Photon & $6.16 \sigma$ \\
$\pi^{0}$ & $($ Combined) \\
$\Lambda$ & \\
Sideband window & $(6.26,6.45) \sigma$ \\
Signal window & $(5.92,6.07) \sigma$ \\
$\Delta \mathrm{E}$ & $(5.77,6.39) \sigma$ \\
$\omega^{\mathrm{bg}}$ & $(6.37,6.41) \sigma$ \\
$M_{\pi^{0} \pi^{0}}$ veto & $(6.06,6.30) \sigma$ \\
\hline \hline
\end{tabular}


We determine the significance of spin- $-\frac{1}{2}$ hypothesis to be $6.16 \sigma$ with this approach.

The systematic uncertainties due to the event selection criteria of the $\Delta E$, signal and sideband events are estimated by varying their requirements by $1 \mathrm{MeV}$. The uncertainty due to the $M_{\pi^{0} \pi^{0}}$ rejection criterion in the $\Lambda_{c}^{+} \rightarrow \Sigma^{+} \pi^{0}$ channel is checked with a tight and loose requirement, i.e., $M_{\pi^{0} \pi^{0}} \in[0.42,0.53] \mathrm{GeV} / c^{2}$ and $[0.38,0.57] \mathrm{GeV} / c^{2}$. The potential bias due to the sideband scale factor, $\omega^{\mathrm{bg}}$, is evaluated through varying the parameters by $1 \sigma$ for the Argus fit function. The ranges of significance estimation are given in Table II. The resulting significance comparing the two hypothesis tests are found to be $6.07 \sigma$ with a systematic boundary $(5.77 \sim 6.45) \sigma$, where the uncertainties correspond to the smallest and largest values listed in Table II.

In conclusion, we have compared the two spin hypotheses $\frac{1}{2}$ and $\frac{3}{2}$ for the $\Lambda_{c}^{+}$baryon by studying the process $e^{+} e^{-} \rightarrow \Lambda_{c}^{+} \bar{\Lambda}_{c}^{-}$, using $587 \mathrm{pb}^{-1}$ of BESIII data collected at $\sqrt{s}=4.6 \mathrm{GeV}$. The analysis considered the joint angular distribution of the production and decay modes $\Lambda_{c}^{+} \rightarrow p K_{S}^{0}, \Lambda \pi^{+}, \Sigma^{0} \pi^{+}$and $\Sigma^{+} \pi^{0}$. We found that the spin of $\frac{1}{2}$ hypothesis is preferred over the $\frac{3}{2}$ with a significance of about $6 \sigma$. Hence, we conclude the spin of the $\Lambda_{c}^{+}$baryon to be $\frac{1}{2}$, consistent with the expectation of the naive quark model. Since the $\Lambda_{c}^{+}$is the lightest charmed baryon, this experimental determination of its spin is also a cornerstone in the extraction of the properties of heavier charmed and beauty baryons whose decay chains include this particle.
The BESIII collaboration thanks the staff of BEPCII and the IHEP computing center for their strong support. This work is supported in part by National Key Basic Research Program of China under Contracts No. 2015CB856700, No. 2020YFA0406300, No. 2020YFA0406400; National Natural Science Foundation of China (NSFC) under Contracts No. 11875262 , No. 11835012 , No. 11625523 , No. 11635010, No. 11735014, No. 11822506, No. 11835012, No. 11935015, No. 11935016, No. 11935018, No. 11961141012; the Chinese Academy of Sciences (CAS) Large-Scale Scientific Facility Program; Joint Large-Scale Scientific Facility Funds of the NSFC and CAS under Contracts No. U2032110, No. U1732263, No. U1832207, No. U1832107; CAS Key Research Program of Frontier Sciences under Contracts No. QYZDJ-SSW-SLH003, No. QYZDJ-SSW-SLH040; 100 Talents Program of CAS; Fundamental Research Funds for the Central Universities; INPAC and Shanghai Key Laboratory for Particle Physics and Cosmology; ERC under Contract No. 758462; German Research Foundation DFG under Contracts Nos. Collaborative Research Center CRC 1044, FOR 2359; Istituto Nazionale di Fisica Nucleare, Italy; Ministry of Development of Turkey under Contract No. DPT2006K-120470; National Science and Technology fund; STFC (United Kingdom); The Knut and Alice Wallenberg Foundation (Sweden) under Contract No. 2016.0157; The Royal Society, UK under Contracts No. DH140054, No. DH160214; The Swedish Research Council; U.S. Department of Energy under Contracts No. DE-FG02-05ER41374, No. DE-SC-0012069; Olle Engkvist Foundation under Contract No. 200-0605.
[1] J. C. Anjos, J. A. Appel, A. Bean, S. B. Bracker, T. E. Browder, L. M. Cremaldi et al. (Tagged Photon Spectrometer Collaboration), Phys. Rev. Lett. 60, 1379 (1988).

[2] P. A. Zyla et al. (Particle Data Group), Prog. Theor. Exp. Phys. (2020), 083C01.

[3] G. F. Chew and S. C. Frautschi, Phys. Rev. Lett. 8, 41 (1962).

[4] H. Y. Cheng, Front. Phys. (Beijing) 10, 101406 (2015).

[5] H. X. Chen, W. Chen, X. Liu, Y. R. Liu, and S. L. Zhu, Rep. Prog. Phys. 80, 076201 (2017).

[6] E. Klempt and J. M. Richard, Rev. Mod. Phys. 82, 1095 (2010).

[7] B. W. Lee and R. F. Sawyer, Phys. Rev. 127, 2266 (1962).

[8] P. D. B. Collins, An Introduction to Regge Theory and High Energy Physics, Cambridge Monographs on Mathematical Physics (Cambridge University Press, Cambridge, England, 2009), p. 133, https://doi.org/10.1017/ CBO9780511897603.

[9] M. Jezabek, K. Rybicki, and R. Rylko, Phys. Lett. B 286, 175 (1992).
[10] M. Gell-Mann, Phys. Lett. 8, 214 (1964).

[11] V. G. Baryshevsky, arXiv:1504.06702.

[12] A. S. Fomin, A. Y. Korchin, A. Stocchi, O. A. Bezshyyko, L. Burmistrov, S. P. Fomin, I. V. Kirillin, L. Massacrier, A. Natochii, P. Robbe et al., J. High Energy Phys. 08 (2017) 120.

[13] E. Bagli, L. Bandiera, G. Cavoto, V. Guidi, L. Henry, D. Marangotto, F. Martinez Vidal, A. Mazzolari, A. Merli, N. Neri et al., Eur. Phys. J. C 77, 828 (2017); 80, 680(E) (2020).

[14] V. G. Baryshevsky, Eur. Phys. J. C 79, 350 (2019).

[15] M. Galanti, A. Giammanco, Y. Grossman, Y. Kats, E. Stamou, and J. Zupan, J. High Energy Phys. 11 (2015) 067.

[16] R. Aaij et al. (LHCb Collaboration), Phys. Rev. Lett. 119, 112001 (2017).

[17] M. Ablikim et al. (BESIII Collaboration), Chin. Phys. C 44, 040001 (2020).

[18] M. Jacob and G. C. Wick, Ann. Phys. (Paris) 7, 404 (1959).

[19] S. U. Chung, CERN Yellow Report No. CERN 71-8, 1971.

[20] H. Chen and R.-G. Ping, Phys. Rev. D 99, 114027 (2019). 
[21] E. Perotti, G. Fäldt, A. Kupsc, S. Leupold, and J. J. Song, Phys. Rev. D 99, 056008 (2019).

[22] M. Ablikim et al. (BESIII Collaboration), Phys. Rev. D 100, 072004 (2019).

[23] M. Ablikim et al., Nucl. Instrum. Methods Phys. Res., Sect. A 614, 345 (2010).

[24] S. Jadach, B. F. L. Ward, and Z. Was, Phys. Rev. D 63, 113009 (2001).

[25] N. Brambilla et al., Eur. Phys. J. C 71, 1534 (2011).

[26] D. J. Lange, Nucl. Instrum. Methods Phys. Res., Sect. A 462, 152 (2001); R. G. Ping, Chin. Phys. C 32, 599 (2008).

[27] S. Agostinelli et al. (GEANT Collaboration), Nucl. Instrum. Methods Phys. Res., Sect. A 506, 250 (2003); J. Allison et al., IEEE Trans. Nucl. Sci. 53, 270 (2006).
[28] M. Ablikim et al. (BESIII Collaboration), Phys. Rev. Lett. 116, 052001 (2016).

[29] H. Albrecht et al. (ARGUS Collaboration), Phys. Lett. B 241, 278 (1990).

[30] F. James and M. Roos, Comput. Phys. Commun. 10, 343 (1975).

[31] T. D. Lee and C. N. Yang, Phys. Rev. 109, 1755 (1958).

[32] R. Aaij et al. (LHCb Collaboration), Phys. Rev. D 92, 011102 (2015).

[33] M. Ablikim et al. (BESIII Collaboration), Phys. Rev. D 89, 071101 (2014).

[34] M. Ablikim et al. (BESIII Collaboration), Phys. Rev. D 92 , 052003(R) (2015); 93, 039906(E) (2015).

[35] M. Ablikim et al. (BESIII Collaboration), Phys. Rev. Lett. 121, 062003 (2018). 